\title{
Research on Safety Protection of Oil Depot Diversion Tunnel
}

\author{
Tang Jian, Zhao Jichang \\ Logistic Engineering University, Chongqing 401311, China
}

Keywords: Oil cave depot, diversion tunnel, safety evaluation, earthquake resistance, reliability

\begin{abstract}
As for the safety of oil depot, the safety of diversion tunnel is very important. In this paper, the safety of oil depot diversion tunnel is regarded as the research object, and the main factors which impact the oil depot diversion tunnel are analyzed. Take the safety of diversion tunnel in the earthquake as an example, the standard working conditions of 8-degree design intensity and III-grade surrounding rock in the earthquake are subject to numerical modeling and calculated by FLAC3D to obtain the displacement of vault and stress value under different buried depth. On this basis, a large number of samples can be obtained by Monte Carlo method, reliability analysis is conducted in two aspects of displacement and stress value, and the reliability of the oil depot diversion tunnel within 0-7 seconds under different buried depth in the earthquake can be calculated. According to the calculation result, the functional effects of diversion tunnel under different buried depth, the major damage nodes during the earthquake, the impact of earthquake stress on the diversion tunnel structure, and the damage effects of earthquake vertical and horizontal waves on the diversion tunnel can be obtained.
\end{abstract}

\section{Introduction}

Safety protection of the oil depot is a complex task. The oil is flammable, explosive, toxic and volatile in storage, and the oil depot has the characteristics of high temperature, high pressure and continuous operation. Oil depot accident occurred frequently in recent years around the world, causing heavy casualties and economic losses. The research on oil depot safety is of great significance for the prevention and reduction of accidents. Most countries take the safety assessment of oil depot as the important basis for site selection of oil depot, accident prevention and the contingency plan.

Oil depot is a kind of oil storage equipment, including vertical cave depot, horizontal cave depot and underground water depot. Any cave depot needs the diversion tunnel to communicate with external devices. This paper takes the oil depot diversion tunnel as the research object. In this paper, the various factors that affect the safety of underground oil depot are analyzed by safety assessment method, in order to find the main factors and study for the solution.

\section{Safety evaluation of oil depot diversion tunnel}

Overview of safety evaluation. Safety assessment is also known as hazard assessment or risk assessment for the purpose of achieving system security, the security system engineering principle and engineering method are adopted to perform qualitative and quantitative analysis of the inherent or potential dangers in the system, the possibility of dangers and the assessment of severity of the consequences can be obtained, and the hazard level of system is obtain in accordance with the evaluation criterion, so the improvement measures can be put forward to decrease the accident rate and losses and obtain the optimal security investment.

Safety assessment has four objectives, namely promoting the realization of inherent safety production, achieving overall process safety control, establishing optimal solution of system security, providing a basis for decision-maker, and achieving standardization and scientization of safety technology and safety management.

Safety evaluation method. In the safety assessment of oil depot, the oil reservoir area safety checklist, work zone safety checklist, bottled warehouse safety checklist and oil pumping station safety checklist are prepared according to different inspection sites. Anti-lightning safety checklist, 
anti-static safety checklist, oil operation safety checklist and storage safety checklist are prepared according to different inspection items.

Basic procedures of safety evaluation, shown in Figure 1:

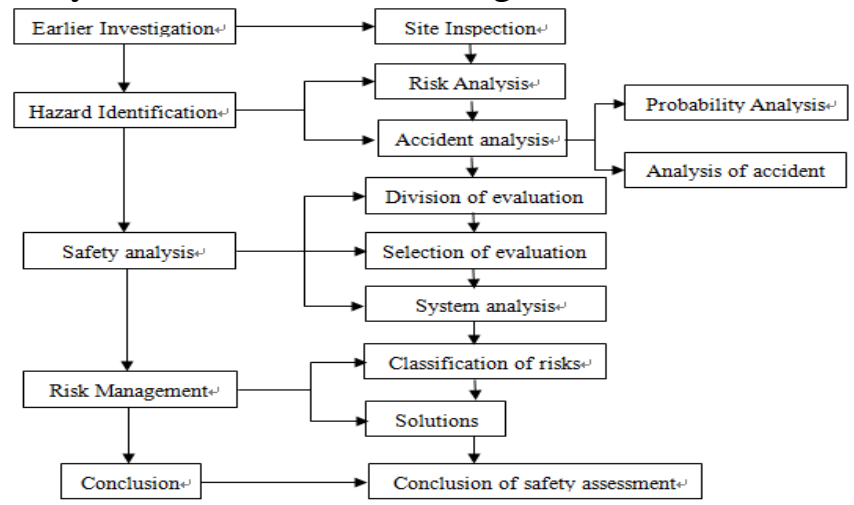

Figure 1 Program chart of safety evaluation

According to the safety evaluation program and in the safety protection of underground oil depot diversion tunnel, the hazard analysis can be conducted in two aspects of design flaw and human factors. The danger of diversion tunnel is usually the explosion, impact and earthquake. This paper analyzes the oil depot in the earthquake the in the rock-soil aspect. Safety assessment of the oil depot is shown in Figure 2.

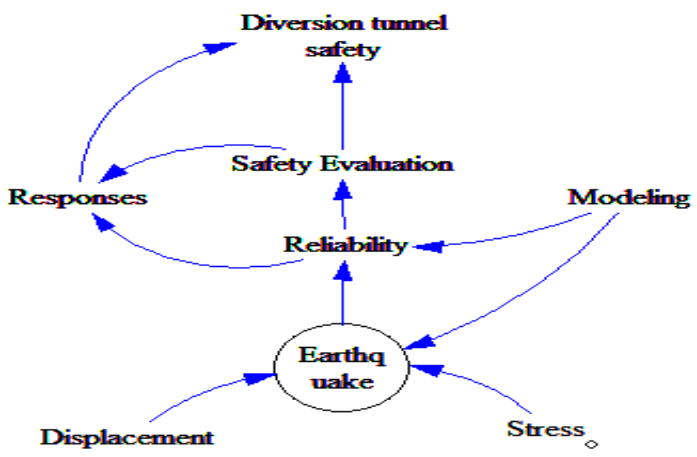

Figure 2 Schematic diagram of safety evaluation of oil cave depot

\section{Anti-seismic model of oil depot diversion tunnel}

Modeling. Take the underground two-lane diversion tunnel as an example, and analyze the response of diversion tunnel in different conditions to determine the performance level. The existing highway tunnel construction specifications [JTJ042-94] show that: under the condition of III-grade or above surrounding rock of the tunnel, when the cladding thickness of diversion tunnel is two times the diameters of the tunnel, the tunnel is stable. In this paper, such working conditions are regarded as the standard conditions, and the diversion tunnel under such working conditions are the standard tunnel for modeling.

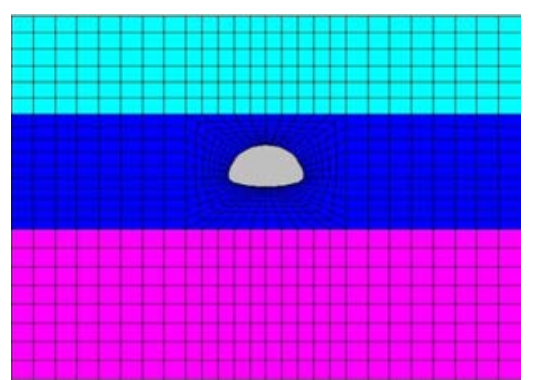

Figure 3 Diversion tunnel model

Constitutive model and material parameter.FLAC3D offers a variety of geotechnical constitutive model, and Moore - Coulomb constitutive model is adopted for realistic simulation of nonlinear mechanical characteristics of rock mass and the lining structures. Model parameters are shown in Table 1. 
Table 1 Seismic Parameters Applied and Tunnel Size Chart

\begin{tabular}{|c|c|c|c|}
\hline $\begin{array}{c}\text { Classification } \\
\text { of surrounding rock }\end{array}$ & III & $\begin{array}{l}\text { Net width } \\
\text { of tunel }\end{array}$ & 12 \\
\hline Density $\rho \quad(\mathrm{Kg} / \mathrm{m} 3)$ & 2550 & Limited height of tunnel & 5 \\
\hline Elasticity modulus $\mathrm{E}$ & 15GPa & $\begin{array}{c}\text { Thickness } \\
\text { of overlying strata }\end{array}$ & 24 \\
\hline Poisson ratio $v$ & 0.25 & Left boundary & 36 \\
\hline Cohesion $C$ & $0.8 \mathrm{MPa}$ & Right boundary & 36 \\
\hline Friction angle $\phi$ & 39 & Lower boundary & 36 \\
\hline
\end{tabular}

Selection of seismic wave. In normal circumstances, the stress deformation of surrounding rock and lining before and after the peak acceleration has great impact on the overall stability. This paper intercepts the fluctuated time-history curve before $7 \mathrm{~s}$ of the EI Centro by filtering software, shown in the figure 4 and figure 5 . The horizontal acceleration peak $0.2 \mathrm{~g}$ (basic seismic intensity is 8 degrees) appears at the 2.1s of the time curve, while the vertical acceleration peak takes $2 / 3$ of the horizontal acceleration peak and appears at the 1s of time curve.

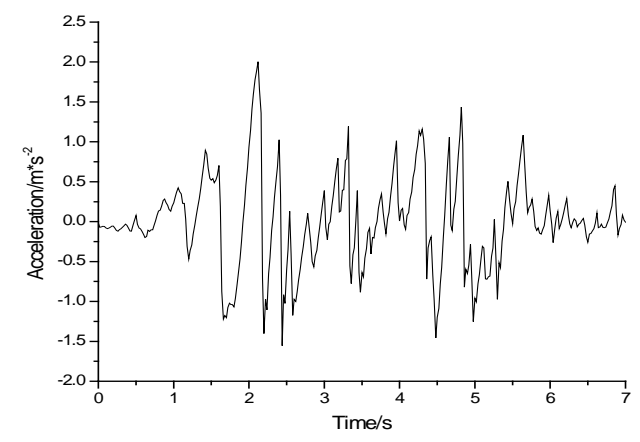

Figure 4 EL Horizontal Wave Pattern

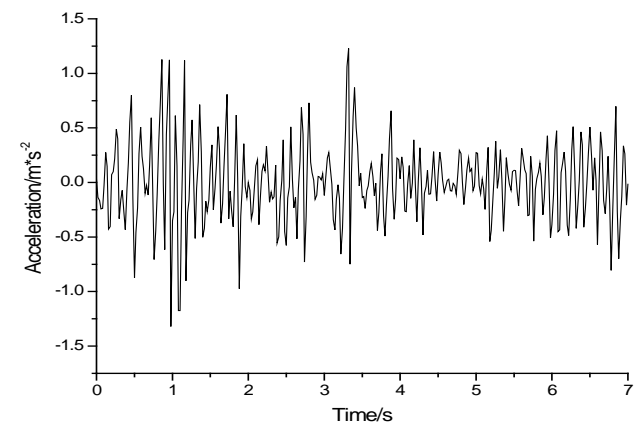

Figure 5 EL longitudinal Wave Pattern

\section{Calculation of reliability}

The basic process of Monte Carlo finite element method is that: First, generate a series of random numbers for each random variable in accordance with uniform distribution. Thus each variable has a set of corresponding random numbers, and each group of random numbers is the sample numbers. Secondly, the random numbers of random variables are individually substituted into the finite element equations to obtain the solution of a set of variables.

In this paper, the parameters are subjected to multiple random samplings to obtain data for calculation.

Anti-seismic reliability analysis based on displacement. The resistant random variable of the structure is $\mathrm{R}$, the random variable of loading effect is $\mathrm{S}$, probability density function is $f_{R}(r)$ and $f_{s}(S)$, the distribution function is $F_{R}(r)$ and $F_{s}(S)$, and R and $\mathrm{S}$ is mutually independent. The failure probability of structure is: 


$$
P_{f}=P(Z<0)=\iint_{r<s} f_{R}(r) f_{s}(S) d r d s
$$

In the standard normalized coordinate system, the shortest distance from the original point to lose efficacy cross section is the reliability index of structure. When $\mathrm{R}$ and $\mathrm{S}$ in the performance function is normally distributed,

$$
P_{f}=\Phi\left(-\frac{\mu_{z}}{\sigma_{z}}\right)=\Phi(-\beta)
$$

In which: $\Phi$ is standard normal distribution function; $\mu_{z}$ and $\sigma_{z}$ is mean value and standard deviation of variable $\mathrm{Z}$; and $\beta$ is the reliability index of structure, and $\beta=\frac{\boldsymbol{\mu}_{z}}{\sigma_{z}}$

The equation (1) can be simplified into secondary moment (mean value and variance) of the random variable.

This paper assumes that if the structure of oil depot exceeds the limiting conditions, the oil depot is damaged. Displacement damage principle is adopted, and the performance function is that:

$$
\boldsymbol{g}=\boldsymbol{u}_{\mathrm{o}}-\boldsymbol{u}_{\max }
$$

In which: $u_{0}$ is maximum displacement specified in the codes; and $u_{\max }$ is the maximum displacement of each structure of the oil depot.

According to the performance function:

$$
P_{f}(g<0)=P\left(u_{\max }>u_{0}\right)=\int_{u_{0}}^{\infty} f(x) d x
$$

The relative displacement of vault in standard tunnel at different depths is drawn as the curves in Figure 6 and Figure 7 by using Flac3D software:

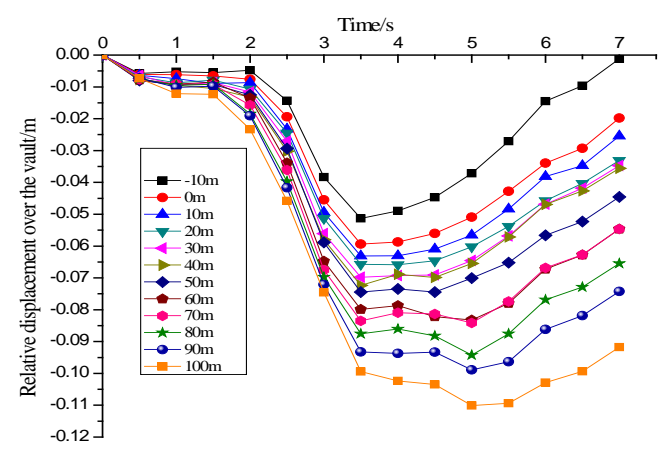

Figure 6 Vertical Relative displacement over the vault under seismic action

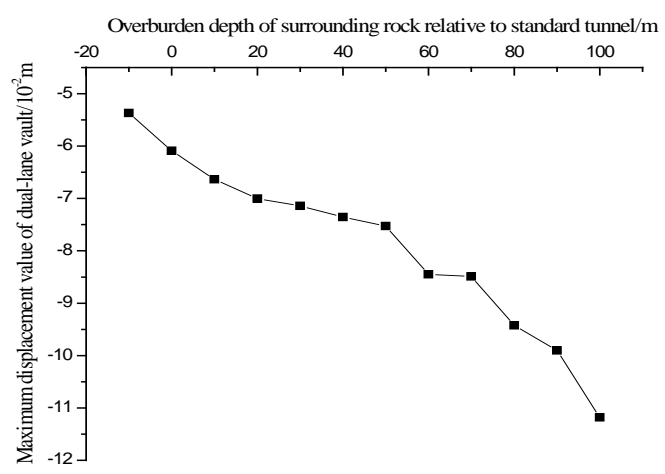

Figure 7 Relationship between overburden depth and maximum displacement over the vault 
Anti-seismic reliability analysis based on ultimate bearing capacity. Meanwhile, the stress of each monitoring point should be considered. This paper uses the existing code (GB50011-2010 "Seismic Design Code"), and the expression of bearing capacity of structural members is as follows:

$$
S \leq \frac{R}{\gamma_{R E}}
$$

In which: S— combination value of self-weight loads and earthquake action

$\mathrm{R}$ — Design value of component bearing capacity

$\gamma_{R E}$ Anti-seismic adjustment coefficient, 0.8

In working condition, the structure may have two states, namely reliability and failure state. Therefore, in the analysis of reliability, for a clearer working condition of the structure and components, it is necessary to know the boundaries of the two states. Figure 11 shows the N-M correlation curves for reinforced concrete columns. Curve ABC is the limiting state . op1 is the bearing capacity, and op2 is the loads suffered. The loads are borne in the area enclosed by OABC, indicating that the bearing capacity of the columns has not reached the limiting conditions and is in a reliable state; the load op2 is on the curve of OABC, indicating that the limiting bearing capacity of the column is in the limiting state; and the load op2 is outside the area enclosed by OABC, indicating that the bearing capacity of the column is insufficient and is in a failure state.

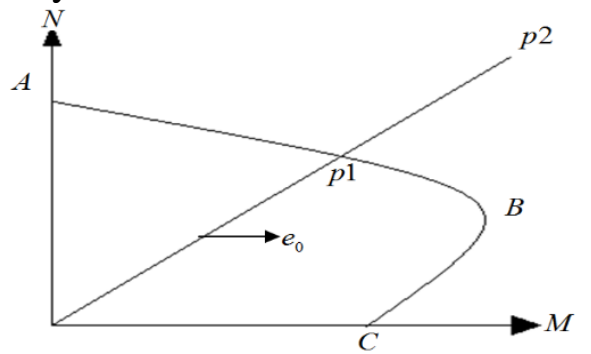

Figure 8 N-M Curve

Limitation equation:

$$
\begin{gathered}
Z=o p 1-o p 2=0 \\
\boldsymbol{p} \mathbf{1}=\sqrt{\left(N_{R}\right)^{2}+\left(\frac{M_{R}}{h}\right)^{2}} \\
o p 2=\sqrt{\left(N_{G S}^{\prime}+N_{E S}^{\prime}\right)^{2}+\frac{\left(M_{G S+}^{\prime} M_{E S}^{\prime}\right)^{2}}{h^{2}}}
\end{gathered}
$$

In which: op1— Resistance of the pillar

op2 _ Action effect of loads

$N_{R} 、 M_{R} \longrightarrow$ pressure and flexural capacity in

h- Height of column cross-section

$N_{G S}^{\prime} 、 M_{G S}^{\prime}$ - Axial force and bending moment of self-possessed loads

$N_{E S}^{\prime} 、 M_{E S}^{\prime} \quad$ Axial force and bending moment caused by earthquake

In the limitation of the above-mentioned bearing capacity, if $\mathrm{Z}>0$, the column is safe; if $\mathrm{Z}=0$, the column is in the limiting condition; and if $\mathrm{Z}<0$, the column is damaged.

The vault stress of standard tunnel in different buried depth is shown as the curve in Figure 9Figure 11 by using Flac3D software: 


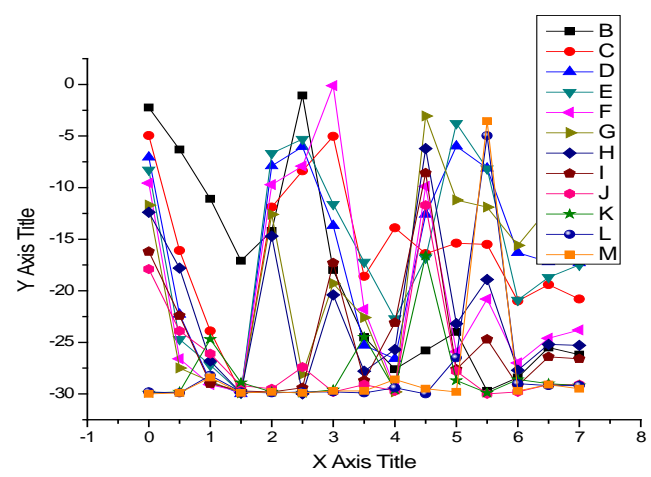

Figure 9 The minimum principal stress

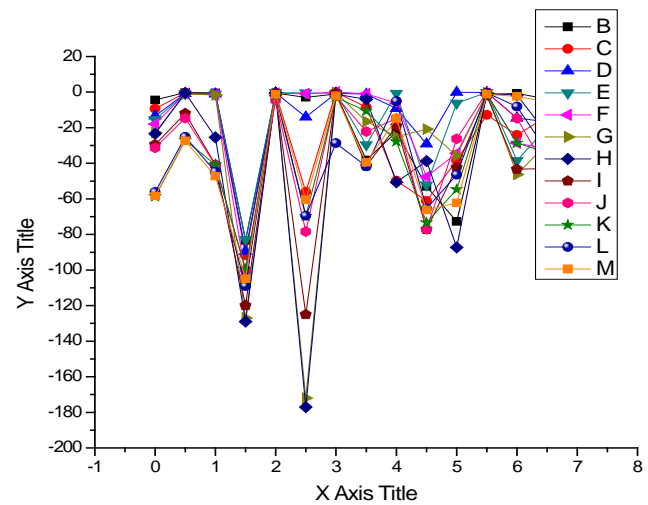

Figure 10 The intermediate principal stress

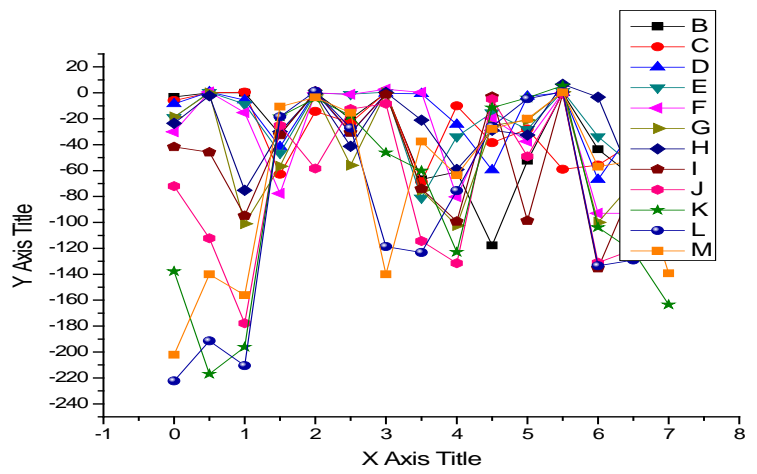

Figure 11 The maximum principal stress

Combining the displacement and stress analysis, the anti-seismic reliability of oil depot diversion tunnel in different depth is calculated. In calculation, if the diversion tunnel is not damaged when the earthquake did not occur, the reliability is 1 ; in order to calculate accurate in reliability, the lowest and deepest limiting conditions are not taken into consideration. The calculation result of anti-seismic reliability of oil depot diversion tunnel in 10m-90m depth is shown in Figure 12 


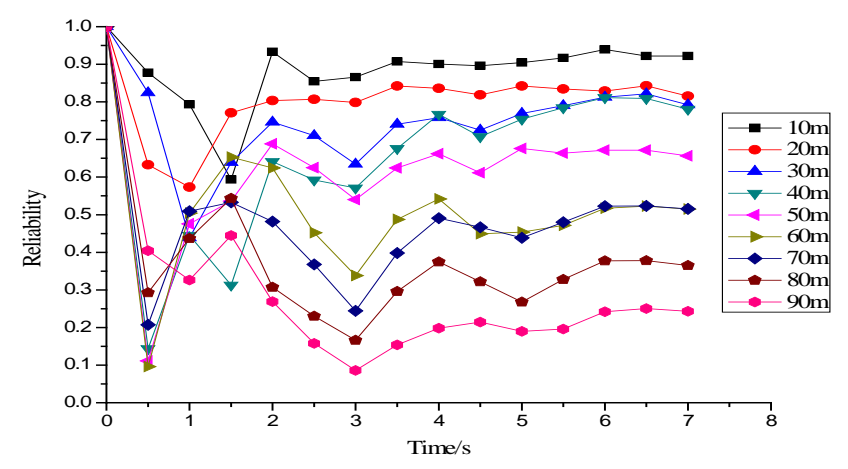

Figure 12 Reliability of oil depot under different buried depth

\section{Conclusion}

The simulation result shows the following conclusion:

(1)When an earthquake occurs, the oil depot diversion tunnel in 5-20m overlaying depth of the standard tunnel is intact in functions; the oil depot diversion tunnel in 20-50m overlaying depth is slightly damaged; the oil depot diversion tunnel in 50-60m overlaying depth is damaged; the oil depot diversion tunnel in 60-80m overlaying depth is greatly damaged; and the oil depot diversion tunnel in over $80 \mathrm{~m}$ overlaying depth is almost collapsed.

(2)When an earthquake occurs, according to the calculation result of vault displacement and stress, the damage to the oil depot diversion tunnel occurs mainly in 2-5 seconds. During this period, the structural reliability of the diversion tunnel is significantly reduced.

(3)The pressure, shear and seismic resistant reliability of the oil depot diversion tunnel is sensitive to the sudden high-load stress. When the earthquake generates high-load stress, the reliability of diversion tunnel structure is sharply decreased.

(4)The calculation result of the reliability shows that the oscillation frequency of secondary wave is more consistent, and the oscillation frequency of primary wave is not related. It is obviously that the secondary wave has greater damage to the diversion tunnel.

\section{Reference}

[1] Chao zhao,Theoretical research on system reliability design of limiting bearing capacity of plan steel-frame structure[D],Master Thesis of Southeast University,2008

[2] Chowdhury R,Rao B N. Probabilities stability assessment of slopes using high dimensional model representation[J]. Computer and Geotechnics, 2010;3(7-8):876-884

[3] Dong Yanqiu, Tian Jingbo, Structural reliability analysis by using Monte Carlo method [J]. Heilongjiang Engineering College Journal, 2010,24(1):27-29

[4] Guo Zhiwei, Bai Guangchen. Application of least squares support vector machine for regression to reliability analysis[J]. Chinese Journal of Aeronauties,2009;22(2):160-166

[5] Gome H M, Awruch A M, Paulo A ML. Reliability based optimization of laminated composite structures using genetic algorithms and artificial neural networks[J]. Structural Safety, 2011, 33(3):186-195

[6] Huang Kun, Zhu Hongjie, Su Xin, Current situation of safety assessment of oil depot [J]. Petroleum Engineering Construction, 2006, 32(1): 1-7

[7] Jian Shuai, Safety Assessment of Crude Oil Depot [J].Chinese Safety Science Journal, 2006, 16(10): 21-24

[8] Liu Yiemin, Zhang Xingkai, Liu Zhigong, Safety Assessment Method Application Guidance [M].Beijing Chemical Industry Press, 2005,4:26 
[9] Ma Xiurang, Design Manual of Oil Depot [M]. China Petrochemical Press, 2009:148

[10] Ma Hongwang, Guofan Zhao, Anti-seismic reliability analysis of reinforced concrete column [J]. Engineering Construction, 2001,31(10):58-61

[11] PRC National Standard, New Standard of Seismic Design of Buildings (GB5001-2010) [S]. Beijing: Chinese Architecture \& Building Press, 2010:241-243

[12] Suryani E, Chou S Y, Hartono R, Chen C H. Demand Scenario Analysis and Planned Capacity Expansion: A System Dynamics Framework[J]. Simulation Modelling Practice and Theory, 2010: 732-751.

[13] Sui Pengcheng, Chen Baozhi, Sui Xu, Safety Principles [M].Beijing: Chemical Industry Press, 2005

[14] Zhu Lili, Research on Finished Oil Depot Safety Assessment[D].Beijing University of Chemical Technology, 2008

[15] Zhu Wanli, Tunneling Construction Technology of Underground Oil Depot Engineering [J], Enterprise Technology Development, 2010,29(12):79-80 